\title{
Multimodal Discourse Analysis of Posters on Wildlife Protection- Based on Visual Grammar
}

\author{
Yue Guan \\ College of Foreign Language, Shandong University of Science and Technology, Qingdao 266590, Shandong Province, China
}

\begin{abstract}
The advent of information age has brought us a plethora of non-text information, such as images, colors, sounds, etc. Consequently, text-based discourse analysis can no longer meet people's demands to obtain the ever-varying information. Due to this reason, multimodal analysis becomes crucial. This paper analyzes 13 public posters on wildlife protection which are collected from the internet. The 13 posters are examined on the basis of three dimensions - represented meaning, interactive meaning and compositional meaning. This study helps poster designers design high-quality posters, as well as assists poster viewers to understand the meanings of public posters on wildlife protection.

Keywords: Multimodal Discourse Analysis; Public Posters on Wildlife Protection; Represented Meaning; Interactive Meaning; Compositional Meaning
\end{abstract}

\section{The introduction of visual grammar}

Multimodal discourse analysis (MDA) has been developed from various aspects since it began to be adopted to analyze multimodal discourse. Kress and Van Leeuwen proposed visual grammar theory and took it as the theoretical framework of MDA in their book, Reading Images: The grammar of visual design. Visual grammar is on the ground of Halliday's Systematic Functional Grammar (SFG) mainly includes three parts, the represented meaning, the interactive meaning as well as the compositional meaning.In terms of the represented meaning, Kress \& Van Leeuwen (2006) hold that any semiotic system has to be able to represent, in a referential or pseudo-referential sense, aspects of the experiential world outside its particular system of signs. (Kress \& Van Leeuwen, 2006: 40).

The interactive meaning is the relationship between the person in posters and outside. There are three kinds of relationships according to contact, social distance and attitude.

The compositional meaning refers to the mutual relationship between in and out posters.

\section{Analysis of posters on wildlife protection}

Posters are the combination of numerous elements, such as color, text, framing and so on. Hence, the analysis of posters must be started from a multimodal perspective.

\subsection{Construction of represented meaning}

Represented meaning is divided into narrative process and conceptual process.

(1) Narrative process

The narrative process is composed of action process, reactional process, speech and mental process. (Kress \&Van Leeuwen, 2006: 63)

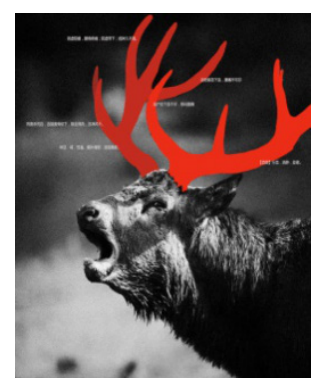

Figure 1. Functions of antlers. (http://zengkai.tuchong.com/2044239/)

\footnotetext{
Copyright (C) 2020 Yue Guan

doi: $10.18282 / 1-$ e.v9i2.1426

This is an open-access article distributed under the terms of the Creative Commons Attribution Non-Commercial License

(http://creativecommons.org/licenses/by-nc/4.0/), which permits unrestricted non-commercial use, distribution, and reproduction in any medium, provided the original work is properly cited.
} 
In Figure 1, the action process is displayed obviously. In this poster, the painful and roaring deer with a pair of red antlers is acted as the actor. The vector is the direction that the deer's head stretches out. The poster describes that a deer is crying at interactive participants who are out of the image. Therefore, there is no goal in this picture. This poster can be depicted as: the deer (actor) is stretching (vector) its head to roar at someone in order to express his enmity.

(2) Conceptual process

Classification process, analytical process and symbolic process consist of the conceptual process.

(i) Analytical process

The carrier and the possessive attribute are expected to figure importantly in the analytical process. The carrier is the whole participants, while the possessive attribute is the detailed components.

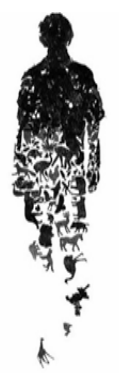

Figure 2. Help reverse climate change before it's too late. (https://huaban.com/pins/1706724264/)

Figure 2 is a typical example. It is a picture of a vanishing man. However, what's the special point of this picture is that the man is composed of diverse fragments of animals. In this picture, the carrier is a man while the possessive attribute is various wild animals which scatter everywhere.

(ii) Symbolic process

Kress and Van Leeuwen say that symbolic process is what a participant means or is. (Kress \& Van Leeuwen, 1996: 105) In this process, the carrier and symbolic attributive have a great influence. The carrier is the participants, while the symbolic attributive is the actual meaning that the participants want to convey. Here, the author takes Figure 3 as an example.

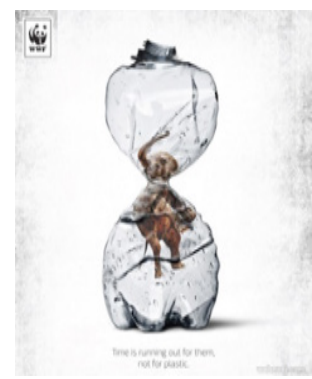

Figure 3. Time is running for them, not for plastic. (http://uuhy.com/html/27469.html?hrtk=123456789)

This is a plastic hourglass. The sign which is used for identifying the symbolic process is the salience of hourglass. In this poster, only the hourglass which is made of the plastic bottle is obvious at the sight of interactive participants. In addition, the hourglass is placed in the middle of this picture. So it is apparent that it is a symbolic process.

\subsection{Construction of interactive meaning}

Interactive meaning represents a kind of social relationship between the represented participants and interactive participants. Contact, social distance and attitude are essential elements in interactive meaning. The following parts will illustrate the detailed information.

(1) Contact

Contact refers to the relationship between inside and outside of the poster. There are two image acts - demand act and offer act. Demand act must have eye line that the represented participants look at the interactive participants directly. While offer act doesn't possess eye-line, it is just that the represented participants expose themselves to interactive participants.

(i) Demand act

Here, the author uses Figure 4 as an example to show a detailed analysis of interactive meaning in public posters on wildlife conservation.

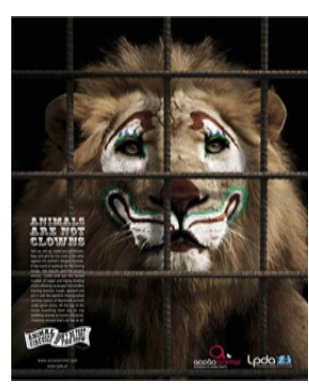

Figure 4. Animals are not Clowns. (http://www.5671.info/hh/image/3396158530/) 
In Figure 4, the lion in this picture which is regarded as represented participant is looking at the interactive participants directly. So the contact is constructed between the lion (represented participant) and poster viewers (interactive participants). In this picture, the lion with a colorful face is locked in an iron cage and looks at the poster viewers directly and piteously. The lion loses freedom.

(ii) Offer act

By contrast, offer act doesn't need eye line between represented participants and poster viewers. The represented participants can convey information by themselves.

(2) Social distance

Social distance can be achieved by means of close shot, medium shot and long shot. It indicates relations between elements in posters and poster viewers.

(i) Close Shot

Here, the author still uses Figure 4 as an example.

This poster is a typical example. Obviously, the intimacy between represented participants and interactive participants increases when they are closer. In Figure 4, it is obvious to see a big lion's head. It almost takes up all the space of the frame. The represented participant is very near to us. So this is close distance.

(ii) Long shot

Long shot shows the impersonal relations between the represented participants and interactive participants. (Wang, 2014: 42) The frame of the camera shows the whole of the represented participant. Here, the author takes Figure 5 as an example.

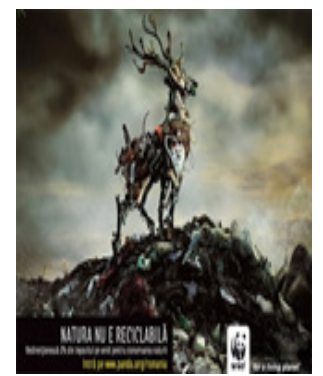

Figure 5. Rubbish can be Recycled, Nature can not. (https://huaban.com/pins/1165390016/)

Figure 5 displays what a long shot is; the main character occupies a little space. In this picture, the deer which is composed of trashes is the represented participants. The deer is showing the whole figure of itself. So it is a long shot distance.

(3) Attitude

Attitude is another way to express the relations between represented participants and interactive participants. It can be classified into horizontal and vertical angle.

Horizontal angle is composed of frontal angle and oblique angle.

As for the oblique angle, Figure 5 is a typical example. The deer that is standing in the trash hill doesn't look at the viewers directly. It looks at somewhere else. So it is oblique angle. The interactive participants feel detached by this oblique angle.

(ii) Vertical angle

The vertical angle includes high angle, eye-eye contact angle and low angle.

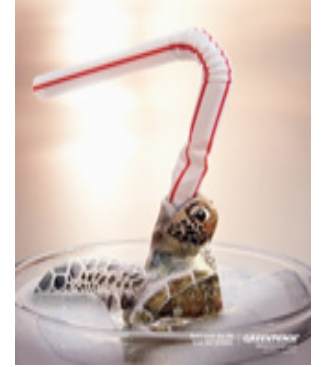

Figure 6. Don't suck the life from our oceans. (https://www-beta1.duitang.com/blog/?id=1005008979)

Figure 6 is a typical example of a high angle. It is obvious to see that the picture is taken by the photographer from a high angle. Then we may know that the interactive participant is standing in a higher position and is empowered to view the represented participant. The poster viewer possesses more power.

In Figure 2, human beings are disappearing gradually with the number of wild animal decreases. There is a metaphor in it. It means that the human beings must pay for eating animals. So this may be taken from a low angle by the photographer. The represented participant (the person) has more power than interactive participant (the viewer) in this poster.

\subsection{Construction of compositional meaning}

Information value, salience and framing are the three principles of compositional meaning. Next, the author will analyze the three elements in detail.

(1) Information value

There are three perspectives to analyze information value. They are left-right, up-down and central-margin. In general, the left is given information, right is new information, up is ideal, down is real, the center is primary and the margin is secondary.

(i) Left-Right 
In general, the left is given information, and the right is ungiven. So the viewer should notice the new information. Here, the author takes Figure 7 as an example.

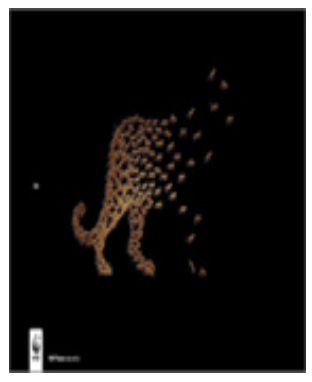

Figure 7. 1984-. (https://www.guokr.com/article/89800/)

In this poster, the left is the year 1984, which is the past year. The number of wild animals is vast. The right part is the future, which is the near future. From Figure 7, the left part is the given messages that everyone knows, while the right is future which viewers cannot forecast.

(ii) Central-margin

The central is important, while the margin is less vital. Here, the author uses Figure 8 as an example.

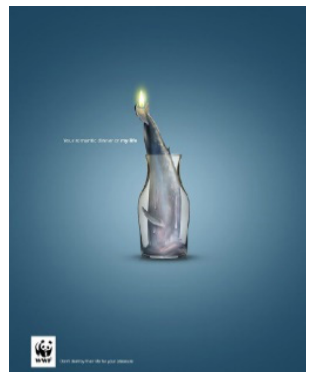

Figure 8. Don't destroy their life for your pleasure. (http:/www.welovead.com/cn/works/details/99fwhqqvm)

Figure 8 is a typical example to prove central-margin information. In Figure 8 , there is a whale fin with a small flare in a bottle and the background is blue. In this picture, the poster designer aims at telling viewers that the whale is in danger by means of putting whale fin in the center. It shows the importance of whales. In Figure 8, the multi-discourse is image and color.

(2) Salience

There are various factors that influence salience, such as size, focus, distance, cultural factors, and so on. Here the author takes Figure 9 as an example.

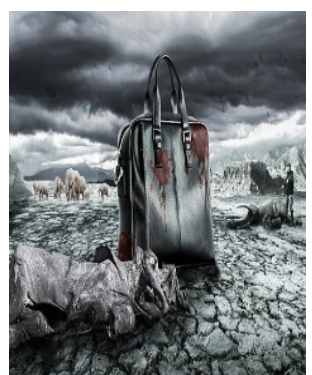

Figure 9. No sale, no kill. (https://www.pinterest.ru/pin/418623727840027016/)

As soon as the viewers see the picture, a bloody bag leaps to the eyes. But why can the bloody bag come into viewers' eyes firstly? The first reason is that the size of this bag is that the biggest in this picture. Another reason is that the red color in this bag which is bright. From the above analysis, it is known that the salience is achieved by focus, size and color. In Figure 9, the multidiscourse is image and color.

\section{Conclusion}

It is understood that the multimodal discourse exists in verbal communication and some other semiotic systems, such as image, color, glance, and so on. The multimodal meaning of these 13 posters on wildlife protection is analyzed under the guidance of visual grammar. Last but not the least, it is important to mention that, unlike the common perception, protecting wildlife is far from being a trivial matter.

\section{References}

1. Halliday, M. A. K. 1985. An Introduction to Functional Grammar [M]. London: Arnold.

2. Kress, G. \& Van Leeuwen, T. 1996. Reading Images: The grammar visual design [M]. London: Routledge.

3. Kress, G. \& Van Leeuwen, T. 2006. Reading Images: The Grammar of Visual Design (2nd edition) [M]. London: Routledge.

4. Silu Wang. Research on the meaning construction of mSultimodal discourse in global warming public welfare posters (in

Chinese). Harbin: Harbin Engineering University; 2014. 